\title{
A GENERAL FAMILY OF CONGRUENCES FOR BERNOULLI NUMBERS
}

\author{
JULIAN ROSEN
}

\begin{abstract}
We prove a general family of congruences for Bernoulli numbers whose index is a polynomial function of a prime, modulo a power of that prime. Our family generalizes many known results, including the von Staudt-Clausen theorem and Kummer's congruence.
\end{abstract}

\section{INTRODUCTION}

1.1. Bernoulli numbers. The Bernoulli numbers $B_{n}$ are rational numbers defined by the power series expansion

$$
\frac{x}{e^{x}-1}=\sum_{n \geq 0} B_{n} \frac{x^{n}}{n !} .
$$

The first few values are:

\begin{tabular}{|c||c|c|c|c|c|c|c|c|c|c|c|c|c|}
\hline$n$ & 0 & 1 & 2 & 3 & 4 & 5 & 6 & 7 & 8 & 9 & 10 & 11 & 12 \\
\hline$B_{n}$ & 1 & $-\frac{1}{2}$ & $\frac{1}{6}$ & 0 & $-\frac{1}{30}$ & 0 & $\frac{1}{42}$ & 0 & $-\frac{1}{30}$ & 0 & $\frac{5}{66}$ & 0 & $-\frac{691}{2730}$ \\
\hline
\end{tabular}

Terms of odd index greater than 1 vanish, and the non-zero terms alternate in sign.

The Bernoulli numbers are known to have interesting arithmetic properties. One well-known example is the von Staudt-Clausen Theorem, which says that for every even integer $n \geq 2$, the quantity

$$
B_{n}+\sum_{\substack{p \text { prime, } \\ p-1 \mid n}} \frac{1}{p}
$$

is an integer. In particular, the denominator of $B_{n}$ is the product of those primes $p$ such that $p-1$ divides $n$. Another well-known result is Kummer's congruence, which says that for all non-negative even integers $m, n$ not divisible by $p-1$, satisfying $m \equiv n \bmod \varphi\left(p^{k}\right)$,

$$
\left(1-p^{m-1}\right) \frac{B_{m}}{m} \equiv\left(1-p^{n-1}\right) \frac{B_{n}}{n} \bmod p^{k}
$$

Date: March 7, 2022. 
Here, a congruence between rational numbers modulo $p^{k}$ means the $p$-adic valuation of their difference is at least $k$ (that is, congruence modulo $\left.p^{k} \mathbb{Z}_{(p)}\right)$. Many generalizations of (1) are known, some involving additional terms, some relaxing the restriction that $p-1 \nmid m$. Several generalizations are given in $[1](\S 9.5, \S 11.4 .2)$

The results of von Staudt-Clausen and Kummer can be expressed in terms Bernoulli numbers whose index is a polynomial in $p$.

(1) Let $f(t) \in \mathbb{Z}[t]$ have positive leading coefficient, and set $\delta=-1$ if $f(1)=0$, and $\delta=0$ if $f(1) \neq 0$. Then the von StaudtClausen theorem implies that

$$
p B_{f(p)} \equiv \delta \quad \bmod p
$$

for every prime $p>|f(1)|$.

(2) Let $f(t), g(t) \in \mathbb{Z}[t]$ be distinct non-constant polynomials with positive leading coefficient, and suppose that $f(1)=g(1) \neq 0$. Let $k$ be the largest power of $t$ dividing $f(t)-g(t)$. Then Kummer's congruence implies that

$$
\frac{B_{f(p)}}{f(p)} \equiv \frac{B_{g(p)}}{g(p)} \bmod p^{k+1}
$$

holds for every prime $p>|f(1)|$.

Here we consider the following problem.

Problem 1. Given polynomials $f_{1}(t), \ldots, f_{n}(t) \in \mathbb{Z}[t]$ with positive leading coefficients, rational functions $g_{0}(t), g_{1}(t), \ldots, g_{n}(t) \in \mathbb{Q}(t)$, and a non-negative integer $N$, determine under which circumstances the congruence

$$
\sum_{i=1}^{n} g_{i}(p) B_{f_{i}(p)} \equiv g_{0}(p) \quad \bmod p^{N}
$$

holds for every sufficiently large prime $p$.

The polynomial form of the von Staudt-Clausen theorem (2) and Kummer's congruence (3) are examples of (4). Other examples are known. For instance, it is a result of Z.-H. Sun [2] that for integers $k$, $b$, with $b \not \equiv 0 \bmod p-1$ even and $k \geq 0$ :

$$
\begin{array}{r}
\frac{B_{k(p-1)+b}}{k(p-1)+b} \equiv k \frac{B_{p-1+b}}{p-1+b}-(k-1)\left(1-p^{b-1}\right) \frac{B_{b}}{b} \bmod p^{2}, \\
\frac{B_{k(p-1)+b} \equiv\left(\begin{array}{c}
k \\
2
\end{array}\right)}{k(p-1)+b} \frac{B_{2(p-1)+b}}{2(p-1)+b}-k(k-2) \frac{B_{p-1+b}}{p-1+b} \\
+\left(\begin{array}{c}
k-1 \\
2
\end{array}\right)\left(1-p^{b-1}\right) \frac{B_{b}}{b} \bmod p^{3} .
\end{array}
$$


A GENERAL FAMILY OF CONGRUENCES FOR BERNOULLI NUMBERS 3

1.2. Results. The main result of this paper is a criterion for (4) to hold.

Theorem 1.1. Fix an integer $N$, non-constant polynomials $f_{1}(t), \ldots, f_{n}(t) \in$ $\mathbb{Z}[t]$ with positive leading coefficients, and rational functions $g_{0}(t), \ldots, g_{n}(t) \in$ $\mathbb{Q}(t)$. Write $v_{t}$ for the $t$-adic valuation on $\mathbb{Q}(t)$, and set

$$
M=\min \left\{v_{t}\left(g_{i}(t)\right)\right\} .
$$

Then the congruence

$$
\sum_{i=1}^{n} g_{i}(p) B_{f_{i}(p)} \equiv g_{0}(p) \quad \bmod p^{N}
$$

holds for every sufficiently large prime $p$ if all of the following conditions hold.

(1)

$v_{t}\left(g_{0}(t)-\left(1-\frac{1}{t}\right) \sum_{\substack{i \\ f_{i}(1)=0}} g_{i}(t)-\sum_{\substack{i \\ f_{i}(1) \geq 2}}\left(1-t^{f_{i}(1)-1}\right) \frac{B_{f_{i}(1)}}{f_{i}(1)} g_{i}(t) f_{i}(t)\right) \geq N$.

(2) For every even, non-positive integer $k \in\left\{f_{i}(1)\right\}$ and every $1 \leq$ $m \leq N-M$

$$
v_{t}\left(\sum_{\substack{i \\ f_{i}(1)=k}} g_{i}(t) f_{i}(t)^{m}\right) \geq N+1-m .
$$

(3) For every even, positive integer $k \in\left\{f_{i}(1)\right\}$ and $2 \leq m \leq$ $N-M$,

$$
v_{t}\left(\sum_{\substack{i \\ f_{i}(1)=k}} g_{i}(t)\left(f_{i}(t)^{m}-k^{m-1} f_{i}(t)\right)\right) \geq N+1-m .
$$

The verification of the conditions (1)-(3) of Theorem 1.1 is a finite computation. The condition that the polynomials $f_{i}(t)$ are nonconstant is for simplicity and is inessential: for each $f_{i}(t)$ that is constant, the term $g_{i}(p) B_{f_{i}(p)}$ can be moved to the right hand side of (7) and made part of $g_{0}(p)$.

Remark 1.2. It can be checked that Theorem 1.1 implies both (2) and (3), so we may view Theorem 1.1 as a common generalization of the von Staudt-Clausen Theorem and Kummer's congruence. The congruences (5) and (6) also follow from Theorem 1.1, as do many of the congruences for Bernoulli numbers given in [1]. 
The author does not know whether the converse of Theorem 1.1 holds. If it does, this is likely quite difficult to prove. In particular, the converse of Theorem 1.1 would imply that there exist infinitely many non-Wolstenholme primes (that is, infinitely many primes $p$ for which $\left.p \nmid B_{p-3}\right)$, which is an open question.

\section{The Kubota-Leopoldt $p$-Adic Zeta Function}

The Riemann zeta function $\zeta(s)$ takes rational values at the nonpositive integers, and we have a formula

$$
\zeta(-n)=-\frac{B_{n+1}}{n+1}
$$

Fix a prime $p \geq 3$. Kummer's congruence implies that the values

$$
\left(1-p^{-s}\right) \zeta(s)
$$

are $p$-adically uniformly continuous if $s$ is restricted to the negative integers in a fixed residue class modulo $p-1$. For $n \geq 2$ an integer, the Kubota-Leopoldt p-adic zeta value is defined by

$$
\zeta_{p}(n):=\lim _{\substack{s \rightarrow n \text {-adically, } \\ s \leq 0 \\ s \equiv n \bmod p-1}}\left(1-p^{-s}\right) \zeta(s) .
$$

The function $n \mapsto \zeta_{p}(n)$ is not $p$-adic analytic, but comes from $p-1$ analytic functions, one for each residue class modulo $p-1$.

The following proposition gives a bound on the valuation of the power series coefficients for the $p$-adic zeta function. It is essentially a repackaging of known results.

Proposition 2.1. Let $p$ be an odd prime, $k$ an even residue class modulo $p-1$. Then there exist coefficients $a_{i}(p, k) \in \mathbb{Q}_{p}$ for $i=0,1,2, \ldots$. such that for every non-negative integer $n$ with $n \equiv k \bmod p-1$, there is a convergent p-adic series identity

$$
B_{n}=\left(1-p^{n-1}\right)^{-1} \sum_{i \geq 0} a_{i}(p, k) n^{i} .
$$

The coefficients $a_{i}(p, k)$ satisfy the following conditions:

$$
a_{0}(p, k)= \begin{cases}1-\frac{1}{p} & \text { if } k \equiv 0 \quad \bmod p-1 \\ 0 & \text { otherwise, }\end{cases}
$$

(2) for all $i, p, k$,

$$
v_{p}\left(a_{i}(p, k)\right) \geq \frac{p-2}{p-1} i-2,
$$


(3) for $p \geq i+2$ and all $k$,

$$
v_{p}\left(a_{i}(p, k)\right) \geq i-1 \text {. }
$$

Proof. Let $\omega_{p}$ be the Teichmüller character. For every residue class $k$ modulo $p-1$, there is a Laurent series expansion for the KubotaLeopoldt $p$-adic $L$-function:

$$
L_{p}\left(s, \omega_{p}^{k}\right)=\sum_{i \geq-1} c_{i, k}(s-1)^{i} .
$$

The relationship between $L_{p}$ and Bernoulli numbers is given by

$$
-\left(1-p^{n-1}\right) \frac{B_{n}}{n}=L_{p}\left(1-n, \omega_{p}^{n}\right),
$$

so we may take $a_{i}(p, k)=(-1)^{i} c_{i-1, k}$. Statement (1) of the Proposition follows form the fact that $L_{p}\left(s, \omega_{p}^{k}\right)$ has a simple pole of residue $1-\frac{1}{p}$ at $s=1$ if $k \equiv 0 \bmod p-1$, and is analytic otherwise.

To get the desired bounds on the valuation of the $a_{i}$, we use [3], Theorem 5.11 (in the case $\chi=\omega_{p}^{k}, f=p, F=p(p-1)$ ):

$L_{p}\left(s, \omega_{p}^{k}\right)=\frac{1}{p(p-1)}(s-1)^{-1} \sum_{j=0}^{\infty} p^{j}(p-1)^{j}\left(\begin{array}{c}1-s \\ j\end{array}\right) B_{j} \sum_{\substack{a=1 \\ p \nmid a}}^{p(p-1)} \frac{\omega_{p}^{k}(a)}{a^{j}}\langle a\rangle^{1-s}$,

where $\langle a\rangle:=a / \omega_{p}(a) \equiv 1 \bmod p$. Write $\langle a\rangle=1+p q_{a}$, with $q_{a} \in \mathbb{Z}_{p}$. We can expand $\langle a\rangle^{s-1}$ as a binomial series to obtain

$L_{p}\left(s, \omega_{p}^{k}\right)=\frac{1}{p(p-1)}(s-1)^{-1} \sum_{i, j=0}^{\infty} p^{i+j}(p-1)^{j}\left(\begin{array}{c}1-s \\ i\end{array}\right)\left(\begin{array}{c}1-s \\ j\end{array}\right) B_{j} \sum_{\substack{a=1 \\ p \nmid a}}^{p(p-1)} \frac{\omega_{p}^{k}(a)}{a^{j}} q_{a}^{i}$.

The innermost summation is $p$-integral, so we conclude that

$$
a_{i}(p, k) \in \frac{1}{p} \sum_{s \geq t \geq i} B_{t} \frac{p^{s}}{s !} \mathbb{Z}_{p}
$$

Now we have $v_{p}\left(B_{t}\right) \geq-1$ by the von Staudt-Claussen Theorem, and $v_{p}(s !) \leq s /(p-1)$, so

$$
v_{p}\left(a_{i}(p, k)\right) \geq \frac{p-2}{p-1} i-2,
$$

which implies statement (2) of the Proposition. Finally, if $p \geq i+2$, then

$$
\frac{p^{s}}{s !} B_{t} \in p^{i} \mathbb{Z}_{p}
$$

for all $s \geq t \geq i$, so in this case we have $v_{p}\left(a_{i}(p, k)\right) \geq i-1$. This completes the proof. 


\section{Proof of the TheOrem}

We start with an easy fact.

Proposition 3.1. Suppose $g(t) \in \mathbb{Q}(t)$ is non-zero. Then

$$
v_{p}(g(p))=v_{t}(g(t))
$$

for all but finitely many primes $p$.

Proof. We can write

$$
g(t)=t^{v_{t}(g(t))} \frac{a(t)}{b(t)}
$$

where $a(t), b(t) \in \mathbb{Z}[t]$ satisfy $a(0) b(0) \neq 0$. The equality (8) then holds for every prime not dividing $a(0) b(0)$.

We are now ready to prove Theorem 1.1.

Proof of Theorem 1.1. Suppose $f_{1}, \ldots, f_{n}$ are non-constant integer polynomials with positive leading coefficient, $g_{0}, \ldots, g_{n}$ are rational functions, and $N$ is an integer, satisfying conditions (1)-(3). We would like to prove that the quantity

$$
A(p):=g_{0}(p)-\sum_{i=1}^{n} g_{i}(p) B_{f_{i}(p)}
$$

is divisible by $p^{N}$ for every sufficiently large prime $p$. We can compute an expression for $A(p)$ in terms of the $a_{i}(p, k)$ from the previous section:

$$
\begin{aligned}
A(p) & =g_{0}(p)-\sum_{k \in \mathbb{Z} /(p-1)} \sum_{\substack{i \\
f_{i}(p) \equiv k(p-1)}} g_{i}(p) B_{f_{i}(p)} \\
& =g_{0}(p)-\sum_{\substack{k \in \mathbb{Z} /(p-1) \\
m \geq 0}}\left(\sum_{\substack{i \\
f_{i}(p) \equiv k(p-1)}}\left(1-p^{f_{i}(p)-1}\right)^{-1} g_{i}(p) f_{i}(p)^{m}\right) a_{m}(p, k) .
\end{aligned}
$$

We have $f_{i}(p) \equiv f_{i}(1)$ modulo $p-1$, so for every $p>\max \left\{f_{i}(1)-f_{j}(1)\right\}$,

$$
A(p)=g_{0}(p)-\sum_{\substack{k \in \mathbb{Z} \\ m \geq 0}}\left(\sum_{\substack{i \\ f_{i}(1)=k}}\left(1-p^{f_{i}(p)-1}\right)^{-1} g_{i}(p) f_{i}(p)^{m}\right) a_{m}(p, k) .
$$

Because $f_{i}(t)$ is non-constant, we have $v_{p}\left(p^{f_{i}(p)}\right) \rightarrow \infty$ as $p \rightarrow \infty$, so

$$
A(p) \equiv g_{0}(p)-\sum_{\substack{k \in \mathbb{Z} \\ m \geq 0}}\left(\sum_{\substack{i \\ f_{i}(1)=k}} g_{i}(p) f_{i}(p)^{m}\right) a_{m}(p, k) \quad \bmod p^{N}
$$


for every sufficiently large prime $p$.

Now, for $p$ larger than $\max \left\{f_{i}(1)\right\}$, we have

$$
a_{0}(p, k)=\left\{\begin{array}{l}
1-\frac{1}{p} \text { if } k=0, \\
0 \text { otherise }
\end{array}\right.
$$

for every $k$ for which the inner sum in (9) is non-empty. This allows us to rewrite (9) using no terms $a_{0}(p, k)$. We can also eliminate the terms $a_{1}(p, k)$ for $k \geq 2$. By Proposition 2.1 we have

$$
B_{k}=\left(1-p^{k-1}\right)^{-1} \sum_{m \geq 0} a_{m}(p, k) k^{m},
$$

so we can solve for $a_{1}(p, k)$ in terms of $a_{m}(p, k), m \geq 2$ :

$$
a_{1}(p, k)=\left(1-p^{k-1}\right) \frac{B_{k}}{k}-\sum_{m \geq 2} a_{m}(p, k) k^{m-1} .
$$

We substitute the expressions for $a_{0}(p, k)$ and $a_{1}(p, k)$ into (9) to obtain

$$
\begin{gathered}
A(p) \equiv g_{0}(p)-\sum_{\substack{k \leq-2 \text { even } \\
m \geq 1}} \sum_{\substack{i \\
f_{i}(1)=k}} g_{i}(p) f_{i}(p)^{m} a_{m}(p, k) \\
-\left(1-\frac{1}{p}\right) \sum_{\substack{i \\
f_{i}(1)=0}} g_{i}(p)-\sum_{m \geq 1} \sum_{\substack{i \\
f_{i}(1)=0}} g_{i}(p) f_{i}(p)^{m} a_{m}(p, 0) \\
-\sum_{k \geq 2 \text { even }}\left(1-p^{k-1}\right) \frac{B_{k}}{k} \sum_{\substack{i \\
f_{i}(1)=k \\
f^{\prime}}} g_{i}(p) f_{i}(p) \\
-\sum_{\substack{k \geq 2 \text { even } \\
m \geq 2}} \sum_{\substack{i \\
f_{i}(1)=k}} g_{i}(p)\left(f_{i}(p)^{m}-k^{m-1} f_{i}(p)\right) a_{m}(p, k) \bmod p^{N} .
\end{gathered}
$$

Finally, by condition (1) of the hypothesis of the theorem,

$$
\begin{gathered}
g_{0}(p)-\left(1-\frac{1}{p}\right) \sum_{\substack{i \\
f_{i}(1)=0}} g_{i}(p) \\
-\sum_{k \geq 2 \text { even }}\left(1-p^{k-1}\right) B_{k} \sum_{\substack{i \\
f_{i}(1)=k}} g_{i}(p) f_{i}(p) \equiv 0 \bmod p^{N} .
\end{gathered}
$$

By condition (2) of the hypothesis of the theorem, for every even $k \leq 0$ and $m \geq 1$,

$$
\sum_{\substack{i \\ f_{i}(1)=k}} g_{i}(p) f_{i}(p)^{m} a_{m}(p, k) \equiv 0 \quad \bmod p^{N}
$$


By condition (3) of the hypothesis of the Theorem, for every even $k \geq 2$ and $m \geq 2$,

$$
\sum_{\substack{i \\ f_{i}(1)=k}} g_{i}(p)\left(f_{i}(p)^{m}-k^{m-1}\right) a_{m}(p, k) \equiv 0 \quad \bmod p^{N} .
$$

We conclude that $A(p) \equiv 0 \bmod p^{N}$ for all but finitely many $p$.

\section{REFERENCES}

[1] Henri Cohen. Number theory. Vol. II. Analytic and modern tools, volume 240 of Graduate Texts in Mathematics. Springer, New York, 2007.

[2] Zhi-Hong Sun. Congruences concerning Bernoulli numbers and Bernoulli polynomials. Discrete Appl. Math., 105(1-3):193-223, 2000.

[3] Lawrence C. Washington. Introduction to cyclotomic fields, volume 83 of Graduate Texts in Mathematics. Springer-Verlag, New York, second edition, 1997. 